\title{
Interdisciplinary Teaching and Research: Challenges and Solutions
}

\author{
Caihong Zhang
}

College of Humanities and Foreign Languages, Xi'an University of Science and Technology, 58

SouthYantaRoad, Xi'an, Shaanxi Province, China

zhangcaihonghappy@163.com

Keywords: Interdisciplinary; Team-teaching; Research; Collaboration

\begin{abstract}
Despite the urgent need of collaboration crossing disciplines in higher education, many challenges still exist, hindering the multidisciplinary work from going efficiently in practice. This essay describes difficulties faced by faculty members in interdisciplinary organizations on teaching and research. It illustrates that the "transaction cost" like lacking support, hard to get promoted in profession etc. for interdisciplinary faculties will be compensated if they get trained, gain diversity recognition and follow the appropriate modes.
\end{abstract}

\section{Introduction}

Cooperation crossing disciplinary boundaries has been gaining increasing support in the last decades in many social organizations like scientific associations, government agencies and industries. However, it was not until the criticizing of the disciplinary mode's limit of producing too much "fragmentation of knowledge" and constraining the "appearance and career success of a relatively large number of interdisciplinary scientists" around the 1970s that higher education institutions had long been structured and formed by the departmental culture where researchers have seldom communication across disciplinary boundaries [1]. Recognizing the promise of interdisciplinary approaches in education, research universities in large numbers launched a campaign of breaking the department barriers, which has transformed the university campus culture, brought about changes in administration level, faculty's professional development, and student cultivation.

In America, two very influential academic associations the NAS (the National Academies of Sciences) and AAU (Association of American Universities) announced their support on education and research crossing disciplines and released reports on how universities facilitated interdisciplinary research. Moreover, more than one hundred research universities have made efforts in interdisciplinary collaboration in the last several decades. Despite these encouraging and positive trends, experimental performances have witnessed some challenges as well as achievement in the process. There is some skepticism about the systematic implementation of crossing disciplinary cooperation in practice given that the interdisciplinary culture has not permeated universities.

\section{Objectives}

Since the concept of interdisciplinary cooperation is mentioned and accepted, lots of higher education institutions have created new majors, minors, education programs etc. accordingly to meet the demand of students [2,3]. The extent of changes made in faculty level has been seldom stressed and overlooked. Even though some changes have been made in administrative leadership to facilitate interdisciplinarity by giving greater autonomy for the interdisciplinary centers or units, issues from the faculty's standing point including granting of joint-appointments, promoting individual professional development and promotion are still remains challengeable and unsolved.

This article aims to comb the difficulties in reality for interdisciplinary faculties in their work and tries to come up with some possible proposals by consulting the experiences of some American universities to facilitate the implementation of crossing disciplinary collaboration in education institutions. 


\section{Challenges Faculty Face in Interdisciplinary Work}

Experimental performances in the research universities in USA have shown that for faculty members who conduct interdisciplinary work in collaboration with colleagues from different department, discipline, or other academic units, there are always "transaction costs" for them to pay. Those may be from the academic level or the intercommunicating level, and the pressures from professional evaluation and promotion because the campus culture tends to undervalue interdisciplinary work [4]. What's more, compared with those work within disciplines, faculty involved in interdisciplinary work is usually facing more challenges, and yet getting less recognition.

Having Few Shared Knowledge Resources in Instructing. In daily teaching, interdisciplinary faculties need to cooperate with scholars from other fields in course designing, course development and teaching so as to meet the demand of students from interdisciplinary background. Compared with those in a certain discipline who have plenty of textbooks, articles, problem sets, visualization packet available, teachers in interdisciplinary center or unit have rare resources to consult or share. They have to spend time negotiating with co-lecturers from course planning to co-teaching. Apart from these, the traditional administrators do not know how to appraise or evaluate them in these efforts.

Being Difficult to Get Recognition and Support in Publishing. Interdisciplinary faculty usually takes part in projects which exceed the boundaries of a single field. Therefore, it is frequent that new research materials, techniques, methods etc. have to be mastered. Yet, considering the scope of the projects, interdisciplinary researchers are often encouraged to pay attention to the breadth of integration and to reduce the complexity of the argument and supporting materials, which, as a result, leads to the fact that the editors of the magazines may think these studies are not deep or strong enough [1]. Besides, because of the crossing-boundary feature, the research results of interdisciplinary faulty cannot completely fit the scope of many journals or academic magazines, making it more difficult to get published compared with those focusing on one discipline.

Many programs interdisciplinary faculties work on may overlap the fields which they are not familiar with but already have specialists. Therefore, it's not easy to communicate and consults these outside scholars very often. Because of all these, it's hard for interdisciplinary to build the close-knit relationship and support in doing research crossing disciplines.

Funds Obtaining Obstacles. The situation in fund application is not promising either. Scholars from interdisciplinary background often cannot be approved of in grant proposals from a certain department or ministry or a discipline because they cannot be classified to this single branch of the discipline or that exact researching field. It's not hard to imagine that most interdisciplinary faculty members do not as productive as their disciplinary colleagues in fund application.

Grim Outlook in Professional Promotion. Studies show that more interdisciplinary research happened through "ad hoc teams" (53\%) than the disciplined research (40\%), less lone research (18\%) than disciplined ones (40\%) [1]. This "ad hoc teams" research mode requires more management skills and is more demanding. However, it is different from the traditional evaluation and reward rules, being unclear of the ownership of the intellectual attribution and property, thus getting less recognition. "lone research" is quite obvious and clear in evaluation.

With the non productive-research record and difficult teaching situation mentioned above, it's almost impossible for the interdisciplinary scholars to meet the standards of evaluation criteria when professional promotion is concerned.

\section{Methods to Reduce the "Transaction Cost"}

Despite those challenges and difficulties for faculty, there are lots of factors which distinguish interdisciplinary mode in education from disciplinary one i.e.: its emphasis on integration and collaboration. By building itself on multi-disciplines and connecting them, interdisciplinary mode tries to solve problems in a new synthetic and unifying way. As Mattson once said, "The interdisciplinary approach can facilitate an orderly, problem-oriented inquiry into complex 
problems." [5]. How to dismantle the boundaries between disciplines to enhance collaboration in problem inquiring and solving for faculties? After all, the prominent and dominant rolls played in any interdisciplinary are the faculties.

Even though there have no detailed guide books for interdisciplinary collaboration, some experimental practices, programs and modes conducted by universities can be consulted to enhance the odd of success for faculty in multidisciplinary environment.

Modes of Interdisciplinary Instructing. For disciplinary faculties, teaching and research are, for most time, isolated, repetitive and lacking of cooperation. Even though many have the experiences of attending seminars, workshops, or team-work to improve their professional development, autonomy associated with their jobs makes instructing easily stuck into individual mode because of the belief that "habits created during the early years will last an academic lifetime" [6]. Whereas, co-teaching and team-teaching are often applied in interdisciplinary instructing

Interdisciplinary organizations like units or centers usually have certain committees responsible for deciding courses according to students' interests and demands. Depending on the contents, several faculty members are chosen for a course. Within them, some are lecturers, someone is leading instructor whose responsibilities including students' exams, grade dispute, projects, and meeting with the teaching assistant regularly for other issues. Then, the faculty members gather together to discuss how to best convey the knowledge to students, how long the course takes, and most importantly, how the team-teaching would be done in detail, and how the course would be developed in a long term. From the collaboration performance at some American universities, team-teaching course usually are instructed in forms of traditional lectures, team-taught lecture lectures, and faculty "panel discussions". In traditional lectures, different faculty members focus on fields of their expertise to provide students with broad landscape of interdisciplinary knowledge. Team-taught lectures focus on certain subjects of the course, while instructors cooperate in providing foundational knowledge. In pane discussions, faculty members highlight important trends in the individual disciplines and come up with solutions for potential problems in the course. These discussion gives faculty chances to show disagreement and mutual respect, know their teaching responsibilities and skills of collaborative instructing. Besides, students will benefit as well because they will be clear about the interrelationship of disciplines on a subject and can get involved in this process rather than receive knowledge passively [7]. This mode of interdisciplinary instructing has lots of advantages over the disciplinary ones. It "blends individual teaching responsibilities with shared teaching, gives faculty the opportunity of communication, mutual learning", and creates vibrant classroom experiences for students [7]. The co/team-teaching style has become a template for interdisciplinary approaches to higher education [8].

Ways of Interdisciplinary Research. As has been mentioned in the beginning of the essay, interdisciplinary faulty faces challenges in conducting crossing disciplinary research like lacking in close relationship, support and trust. Moreover, it's not easy for interdisciplinary scholars to get recognition in publishing and evaluations. However, the many merits interdisciplinary collaboration have offset these defects. Here are some key characteristics most cooperation teams share.

First, the scope of the research usually is multi-disciplinary but the width of it should be "focused and small enough" to be "representative", and at the same time be "large enough to be highly relevant" [9]. When composing essays, interdisciplinary faculty need to be careful to use knowledge, methods, language etc. crossing disciplines, and make sure to suggest reviewers who are "broadly familiar with relevant multiple literatures" and have an "interdisciplinary orientation" $[1,9]$.

Second, to bridge the gap brought about by different disciplines, faculties must get trained appropriately to develop "cultural competence", so that they can identify the common ground crossing disciplines, recognize the diversity and be willing to invest time and energy to boost cooperation with members from other disciplines in team work.

Third, integration is the key point in interdisciplinary research which connects the different members from different disciplines to solve certain problems. So the team or organization requires a 
clear approach, method and framework to "mobilize the disciplines" into a "common endeavor" to problems addressing [9].

\section{Conclusion}

Interdisciplinary faculties need to be creative to apply diverse strategies to face the many challenges and overcome the difficulties in teaching, research and interpersonal communication. In the Meantime, the long-established campus culture and rules of educational institutions have to be changed also to pave ways for the professional development of interdisciplinary faculties so that collaboration can be undertaken efficiently.

\section{Acknowledgements}

This article is funded by Shaanxi Soft Science Research Program (2015KRM129).

\section{References}

[1] G. Susan Clark etc. Professional development of interdisciplinary environmental scholars. Journal of Environment Study Science (1), (2011), 99-113.

[2] S. Vincent. Trends in interdisciplinary environmental and sustainability education. AASHE Conference, October, 2012.

[3] S. Vincent, S. Bunn and L. Sloane Interdisciplinary Environmental and Sustainability Education on the Nation's. 2013.

[4] M. Sa. Creso. Interdisciplinary strategies' in U.S. research universities. High Education 55 (2008) 537-552.

[5] D.J. Mattson, S.G. Clark, The Human Dignity in Concept and Practice. Policy Sci (in press)

[6] N. Mills. Now that I'm tenured, where do I go from here? The vitality of mid-career faculty. Council on Undergraduate Research Quarterly, 20, (2000)181-183.

[7] Lisa Benton-Short, Kathleen A. Merrigan. Beyond interdisciplinary: how sustainability create support unities for pan-university efforts. J Environ Stud Sci. (2015). Published on line.

[8] P. Barlett, Chase GW Curricular innovation for sustainability: the Piedmont/Ponderosa model of faculty development. Lib Educ 98(4) (2012):14-21.

[9] SM. Reich, JA. Reich. Cultural competence in interdisciplinary collaborations: A method for respecting diversity in research partnerships. Am J Commun Psychol (38) (2006):51-62. 